\title{
ON FIXED POINT RESULT IN DOUBLE CONTROLLED METRIC SPACES
}

\author{
S.S.P. Singh \\ Assistant Professor, Department of Mathematics, S.N. Sinha College, Warisaliganj, Nawada, Bihar
}

\begin{abstract}
In this paper, we introduce Reich type contractions in the class of double controlled metric spaces and establish fixed point theorem. Our results are generalization of some known results of literature. We also provide example to illustrate significance of the established result.
\end{abstract}

Keywords - Fixed point, a double controlled type metric, controlled type metric, extended b - metric space.

\section{INTRODUCTION}

The notion of a b - metric spaces was studied by Bakhtin [ 1 ], Czerwik [2] and many fixed point results were obtained for single and multivalued mappings by Czerwik and many other authors. ( see [ 3 ]-[ 8 ]) The generalizations of $b$ metric spaces Kamran et al. [ 9 ] and others ( see [ 10 ]-[ 13 ] ) was introduced extended b - metric spaces by controlling the triangle inequality rather than using control function in the contractive conditions. Proving extensions of Banach contraction principle from metric spaces to $b$ - metric spaces and hence to controlled metric type spaces. Recent article [11 ] introduced double controlled metric type spaces and prove Banach Contraction Principle and Kannan [ 14 ] type contraction in double controlled metric type spaces which is generalized the results of [ 12 ], [ 13 ].

In this paper we first define Reich type contractions [ 15,16 ] in the setting of double controlled metric type spaces and prove fixed point results. We also provide example to illustrate significance of the established result.

Definition 1.1 [ 9 ] Given a function $\theta: X \times X \rightarrow[1, \infty)$, where $X$ is a nonempty set. The function $p: X \times X \rightarrow[0, \infty$ ) is called an extended $b$ - metric if

1. $P(x, y)=0$ if and only if $x=y$,

2. $P(x, y)=p(y, x)$

3. $P(x, y) \leq \theta(x, y)[p(x, z)+p(z, y)]$, for all $x, y, z \varepsilon X$.

Mlaiki et al. [ 11 ] generalized the notion of $b$ - metric spaces.

Definition 1.2 [ 11 ] Given a function $\alpha: X \times X \rightarrow[1, \infty)$, where $X$ is a nonempty set. Let function $q: X \times X \rightarrow[0, \infty$ ). Suppose that

1. $q(x, y)=0$ if and only if $x=y$,

2. $\mathrm{q}(\mathrm{x}, \mathrm{y})=\mathrm{q}(\mathrm{y}, \mathrm{x})$,

3. $\mathrm{q}(\mathrm{x}, \mathrm{y}) \leq \alpha(\mathrm{x}, \mathrm{z}) \mathrm{q}(\mathrm{x}, \mathrm{z})+\alpha(\mathrm{z}, \mathrm{y}) \mathrm{q}(\mathrm{z}, \mathrm{y})$, for all $\mathrm{x}, \mathrm{y}, \mathrm{z} \varepsilon \mathrm{X}$.

Then $\mathrm{q}$ is called a controlled metric type and $(X, q)$ is called a controlled metric type spaces.

Now, we introduce a more general b - metric space.

Definition 1.3 [ 12 ] Given non-comparable functions $\alpha, \beta: X \times X \rightarrow[1, \infty)$. The function $\mathrm{d}: \mathrm{X} \times \mathrm{X} \rightarrow[0, \infty)$ satisfies

1. $d(x, y)=0$ if and only if $x=y$,

2. $d(x, y)=d(y, x)$

3. $d(x, y) \leq \alpha(x, z) d(x, z)+\beta(z, y) d(z, y)]$, for all $x, y, z \in X$.

Then $\mathrm{d}$ is called a double controlled metric type by $\alpha$ and $\beta$.

Remark 1.1 A controlled metric type is also a double controlled metric type when taking the same function. The converse is not true in general.

Example 1.1 [ 12 ] Let $X=\{0,1,2\}$. Consider the double controlled type metric

$\mathrm{d}: \mathrm{X} \times \mathrm{X} \rightarrow[0, \infty)$ defined by

$\mathrm{d}(0,0)=\mathrm{d}(1,1)=\mathrm{d}(2,2)=0, \mathrm{~d}(0,1)=\mathrm{d}(1,0)=1, \mathrm{~d}(0,2)=\mathrm{d}(2,0)=1 / 2, \mathrm{~d}(1,2)=\mathrm{d}(2,1)=2 / 5$

and $\alpha, \beta: \mathrm{X} \times \mathrm{X} \rightarrow[1, \infty)$ defined by

$\alpha(0,0)=\alpha(1,1)=\alpha(2,2)=\alpha(0,2)=\alpha(2,0)=1, \alpha(0,1)=\alpha(1,0)=11 / 10$,

$\alpha(1,2)=\alpha(2,1)=8 / 5$. 
$\beta(0,0)=\beta(1,1)=\beta(2,2)=1, \beta(0,1)=\beta(1,0)=11 / 10, \beta(0,2)=\beta(2,0)=3 / 2$, $\beta(1,2)=\beta(2,1)=5 / 4$

Note that, $\mathrm{d}(0,1)>\alpha(0,2) \mathrm{d}(0,2)+\alpha(2,1) \mathrm{d}(2,1)$.

Thus $\mathrm{d}$ is not a controlled metric type for the function $\alpha$.

Definition 1.4 [ 12 ] Let ( X, d ) be a double controlled metric type spaces by one or two functions

1. The sequence $\left\{x_{n}\right\}$ is convergent to some $x \varepsilon X$, if for each positive $\varepsilon$, there is some integer $N$ such that $d\left(x_{n}, x\right)<$ $\varepsilon$ for each $n \geq N$. It is also written as $\lim _{n \rightarrow \infty} x_{n}=x$.

2. The sequence $\left\{x_{n}\right\}$ is said Cauchy if for every $\varepsilon>0, d\left(x_{n}, x_{m}\right)<\varepsilon$ for all $m, n \geq N$, where $N$ is some integer .

3. ( $X, d)$ is said complete if every Cauchy sequence is convergent.

Definition 1.5 [ 12 ] ] Let ( X, d ) be a double controlled metric type spaces by either one function or two functions for $\mathrm{x} \varepsilon \mathrm{X}$ and $\mathrm{k}>0$.

1. We define $\mathrm{B}(\mathrm{x}, \mathrm{k})$ as

$$
\mathrm{B}(\mathrm{x}, \mathrm{k})=\{\mathrm{y} \varepsilon \mathrm{X}, \mathrm{d}(\mathrm{x}, \mathrm{y})<\mathrm{k} .\}
$$

2. The self map $T$ on $X$ is said to be continuous at $\mathrm{X}$ in $\mathrm{X}$ if for all $\delta>0$, there exists $\mathrm{k}>0$ such that $\mathrm{T}(\mathrm{B}(\mathrm{x}, \mathrm{k})) \mathrm{C} \mathrm{B}(\mathrm{Tx}, \delta)$.

Note that if $T$ is continuous at $\mathrm{x}$ in $(\mathrm{X}, \mathrm{d})$, then $\mathrm{x}_{\mathrm{n}} \rightarrow \mathrm{x}$ implies that $\mathrm{Tx}_{\mathrm{n}} \rightarrow \mathrm{Tx}$, when $n \rightarrow \infty$.

\section{METHODOLOGY}

Result on Reich type contractions.

Theorem 2.1 Let $(X, d$ ) be a double controlled metric type spaces. Let $T: X \rightarrow X$ be function, so that there $a, b, c \varepsilon$ $(0,1)$ with $\mathrm{k}=\mathrm{a}+\mathrm{b} / 1-\mathrm{c}<1$,

$\mathrm{d}(\mathrm{Tx}, \mathrm{Ty}) \leq \mathrm{ad}(\mathrm{x}, \mathrm{y})+\mathrm{bd}(\mathrm{x}, \mathrm{Tx})+\mathrm{cd}(\mathrm{y}, \mathrm{Ty})$

for all $\mathrm{x}, \mathrm{y} \varepsilon \mathrm{X}$. For $\mathrm{x}_{0} \varepsilon \mathrm{X}$, take $\mathrm{x}_{\mathrm{n}}=\mathrm{T}^{\mathrm{n}} \mathrm{X}_{0}$.

Assume that,

$\operatorname{Sup}_{\mathrm{m}} \geq 1 \beta\left(\mathrm{x}_{\mathrm{i}+1}, \mathrm{x}_{\mathrm{m}}\right) \quad \alpha\left(\mathrm{x}_{\mathrm{i}+1}, \mathrm{x}_{\mathrm{i}+2}\right) / \alpha\left(\mathrm{x}_{\mathrm{i}}, \mathrm{x}_{\mathrm{i}+1}\right)<1 / \mathrm{k}$.

Suppose that $\lim _{n \rightarrow \infty} \alpha\left(x_{n}, x\right)$ and $\lim _{n \rightarrow \infty} \beta\left(x, x_{n}\right)$ exist are finite and

2 for every $\mathrm{x} \& \mathrm{X}$, then $\mathrm{X}$ passes a unique fixed point.

Proof - The considered sequence $\left\{x_{n}\right\}$ verifies $x_{n+1}=T_{n}$ for all $n \varepsilon N$. Obviously, if there exists $n_{0} \varepsilon N$ for which $\mathrm{x}_{\mathrm{n} 0+1}=\mathrm{x}_{\mathrm{n} 0}$, then $\mathrm{Tx}_{\mathrm{n} 0}=\mathrm{x}_{\mathrm{n} 0}$ and the proof is finished. Thus, we suppose that

$\mathrm{X}_{\mathrm{n}+1} \neq \mathrm{X}_{\mathrm{n}}$ for every $\mathrm{n} \varepsilon \mathrm{N}$. Thus, by ( 1 ), we have

$$
\begin{aligned}
\mathrm{d}\left(\mathrm{x}_{\mathrm{n}}, \mathrm{x}_{\mathrm{n}+1}\right)= & \mathrm{d}\left(\mathrm{Tx}_{\mathrm{n}-1}, \mathrm{Tx}_{\mathrm{n}}\right) \\
\leq & \mathrm{ad}\left(\mathrm{x}_{\mathrm{n}-1}, \mathrm{x}_{\mathrm{n}}\right)+\mathrm{bd}\left(\mathrm{x}_{\mathrm{n}-1}, \mathrm{Tx}_{\mathrm{n}-1}\right)+\mathrm{cd}\left(\mathrm{x}_{\mathrm{n}}, \mathrm{Tx}_{\mathrm{n}}\right) \\
& =\mathrm{ad}\left(\mathrm{x}_{\mathrm{n}-1}, \mathrm{x}_{\mathrm{n}}\right)+\mathrm{bd}\left(\mathrm{x}_{\mathrm{n}-1}, \mathrm{x}_{\mathrm{n}}\right)+\mathrm{cd}\left(\mathrm{x}_{\mathrm{n}}, \mathrm{x}_{\mathrm{n}+1}\right) \\
(1-\mathrm{c}) \mathrm{d}\left(\mathrm{x}_{\mathrm{n}}, \mathrm{x}_{\mathrm{n}+1}\right) & \leq(\mathrm{a}+\mathrm{b}) \mathrm{d}\left(\mathrm{x}_{\mathrm{n}-1}, \mathrm{x}_{\mathrm{n}}\right) \\
\mathrm{d}\left(\mathrm{x}_{\mathrm{n}}, \mathrm{x}_{\mathrm{n}+1}\right) & \leq \mathrm{a}+\mathrm{b} / 1-\mathrm{cd}\left(\mathrm{x}_{\mathrm{n}-1}, \mathrm{x}_{\mathrm{n}}\right) \\
\mathrm{d}\left(\mathrm{x}_{\mathrm{n}}, \mathrm{x}_{\mathrm{n}+1}\right) & \leq \mathrm{kd}\left(\mathrm{x}_{\mathrm{n}-1}, \mathrm{x}_{\mathrm{n}}\right) .
\end{aligned}
$$

Thus, we have

$$
\begin{aligned}
\mathrm{d}\left(\mathrm{x}_{\mathrm{n}}, \mathrm{x}_{\mathrm{n}+1}\right) & \leq \mathrm{kd}\left(\mathrm{x}_{\mathrm{n}-1}, \mathrm{x}_{\mathrm{n}}\right) \\
& \leq \mathrm{k}^{2} \mathrm{~d}\left(\mathrm{x}_{\mathrm{n}-2}, \mathrm{x}_{\mathrm{n}-1}\right) \\
& \leq \ldots \\
& \leq \mathrm{k}^{\mathrm{n}} \mathrm{d}\left(\mathrm{x}_{0}, \mathrm{x}_{1}\right)
\end{aligned}
$$

For all $n, m \varepsilon N(n<m)$, we have

$$
\begin{aligned}
\mathrm{d}\left(\mathrm{x}_{\mathrm{n}}, \mathrm{x}_{\mathrm{m}}\right) \leq \alpha\left(\mathrm{x}_{\mathrm{n}}, \mathrm{x}_{\mathrm{n}+1}\right) \mathrm{d}\left(\mathrm{x}_{\mathrm{n}}, \mathrm{x}_{\mathrm{n}+1}\right)+\beta\left(\mathrm{x}_{\mathrm{n}+1}, \mathrm{x}_{\mathrm{m}}\right) \mathrm{d}\left(\mathrm{x}_{\mathrm{n}+1}, \mathrm{x}_{\mathrm{m}}\right) \\
\leq \alpha\left(\mathrm{x}_{\mathrm{n}}, \mathrm{x}_{\mathrm{n}+1}\right) \mathrm{d}\left(\mathrm{x}_{\mathrm{n}}, \mathrm{x}_{\mathrm{n}+1}\right)+\beta\left(\mathrm{x}_{\mathrm{n}+1}, \mathrm{x}_{\mathrm{m}}\right) \alpha\left(\mathrm{x}_{\mathrm{n}+1}, \mathrm{x}_{\mathrm{n}+2}\right) \\
\mathrm{d}\left(\mathrm{x}_{\mathrm{n}+1}, \mathrm{x}_{\mathrm{n}+2}\right)+\beta\left(\mathrm{x}_{\mathrm{n}+1}, \mathrm{x}_{\mathrm{m}}\right) \beta\left(\mathrm{x}_{\mathrm{n}+2}, \mathrm{x}_{\mathrm{m}}\right) \mathrm{d}\left(\mathrm{x}_{\mathrm{n}+2}, \mathrm{x}_{\mathrm{m}}\right) \\
\leq \alpha\left(\mathrm{x}_{\mathrm{n}}, \mathrm{x}_{\mathrm{n}+1}\right) \mathrm{d}\left(\mathrm{x}_{\mathrm{n}}, \mathrm{x}_{\mathrm{n}+1}\right)+\beta\left(\mathrm{x}_{\mathrm{n}+1}, \mathrm{x}_{\mathrm{m}}\right) \alpha\left(\mathrm{x}_{\mathrm{n}+1}, \mathrm{x}_{\mathrm{n}+2}\right) \\
\mathrm{d}\left(\mathrm{x}_{\mathrm{n}+1}, \mathrm{x}_{\mathrm{n}+2}\right)+\beta\left(\mathrm{x}_{\mathrm{n}+1}, \mathrm{x}_{\mathrm{m}}\right) \beta\left(\mathrm{x}_{\mathrm{n}+2}, \mathrm{x}_{\mathrm{m}}\right) \alpha\left(\mathrm{x}_{\mathrm{n}+2}, \mathrm{x}_{\mathrm{n}+3}\right) \mathrm{d}\left(\mathrm{x}_{\mathrm{n}+2}, \mathrm{x}_{\mathrm{n}+3}\right)
\end{aligned}
$$$$
\leq \ldots
$$$$
+\beta\left(x_{n+1}, x_{m}\right) \beta\left(x_{n+2}, x_{m}\right) \beta\left(x_{n+3}, x_{m}\right) d\left(x_{n+3}, x_{m}\right)
$$

$\leq \alpha\left(\mathrm{x}_{\mathrm{n}}, \mathrm{x}_{\mathrm{n}+1}\right) \mathrm{d}\left(\mathrm{x}_{\mathrm{n}}, \mathrm{x}_{\mathrm{n}+1}\right)+\sum_{i=n+1}^{m-2}\left(\prod_{j=n+1}^{i} \beta\left(x_{j}, x_{m}\right)\right) \alpha\left(x_{i}, x_{i+1}\right) \mathrm{d}\left(x_{i}, x_{i+1}\right)+\prod_{k=n+1}^{m-1} \beta\left(x_{k}, x_{m}\right) d\left(x_{m-1}, x_{m}\right)$

$\leq \alpha\left(\mathrm{x}_{\mathrm{n}}, \mathrm{x}_{\mathrm{n}+1}\right) \mathrm{k}^{\mathrm{n}} \mathrm{d}\left(\mathrm{x}_{0}, \mathrm{x}_{1}\right)+\sum_{i=n+1}^{m-2}\left(\prod_{j=n+1}^{i} \beta\left(x_{j}, x_{m}\right)\right) \alpha\left(x_{i}, x_{i+1}\right) k^{i} d\left(x_{0}, x_{1}\right)+\prod_{k=n+1}^{m-1} \beta\left(x_{k}, x_{m}\right) k^{m-1} d\left(x_{0}, x_{1}\right)$

$$
5
$$

$\leq \alpha\left(\mathrm{x}_{\mathrm{n}}, \mathrm{x}_{\mathrm{n}+1}\right) \mathrm{k}^{\mathrm{n}} \mathrm{d}\left(\mathrm{x}_{0}, \mathrm{x}_{1}\right)+\sum_{i=n+1}^{m-1}\left(\prod_{j=0}^{i} \beta\left(x_{j}, x_{m}\right)\right) \alpha\left(x_{i}, x_{i+1}\right) \mathrm{k}^{\mathrm{i}} d\left(x_{0}, x_{1}\right) \ldots \ldots$

Let $\mathrm{S}_{\mathrm{l}}=\sum_{i=0}^{l}\left(\prod_{j=0}^{i} \beta\left(x_{j}, x_{m}\right)\right) \alpha\left(x_{i}, x_{i+1}\right) k^{i} d\left(x_{0}, x_{1}\right) \ldots \ldots$. 


\section{International Advanced Research Journal in Science, Engineering and Technology}

Vol. 8, Issue 6, June 2021

DOI: $10.17148 /$ IARJSET.2021.86112

Consider

$\mathrm{V}_{\mathrm{i}}=\prod_{j=0}^{i} \beta\left(x_{j}, x_{m}\right) \alpha\left(x_{i}, x_{i+1}\right) \mathrm{k}^{\mathrm{i}} d\left(x_{0}, x_{l}\right)$

We have

$\mathrm{V}_{\mathrm{i}+1} / \mathrm{V}_{\mathrm{i}}=\beta\left(\mathrm{x}_{\mathrm{i}+1}, \mathrm{x}_{\mathrm{m}}\right) \alpha\left(\mathrm{x}_{\mathrm{i}+1}, \mathrm{x}_{\mathrm{i}+2}\right) \mathrm{k} / \alpha\left(\mathrm{x}_{\mathrm{i}}, \mathrm{x}_{\mathrm{i}+1}\right) \ldots \ldots \ldots$

In view of condition (2) and the ratio test, we ensure that the series $\sum v_{i}$ converges. Thus, $\lim _{n \rightarrow \infty} S_{n}$ exists. Hence, the real sequence $\left\{S_{n}\right\}$ is Cauchy.

Now, using (5), we get

$\mathrm{d}\left(\mathrm{x}_{\mathrm{n}}, \mathrm{x}_{\mathrm{m}}\right) \leq \mathrm{d}\left(\mathrm{x}_{0}, \mathrm{x}_{1}\right)\left[\mathrm{k}^{\mathrm{n}} \alpha\left(\mathrm{x}_{\mathrm{n}}, \mathrm{x}_{\mathrm{n}+1}\right)+\left(\mathrm{S}_{\mathrm{m}-1}-\mathrm{S}_{\mathrm{n}}\right)\right]$.

Above, we used $\alpha(\mathrm{x}, \mathrm{y}) \geq 1$. Letting $\mathrm{n}, \mathrm{m} \rightarrow \infty$ in $(10)$ we obtain

$\operatorname{Lim}_{n, m \rightarrow \infty} d\left(x_{n}, x_{m}\right)=0$.

Thus, the sequence $\left\{x_{n}\right\}$ is Cauchy in the complete double controlled metric space $(X, d)$. So, there is some $x^{*} \varepsilon X$, so that

$$
\lim _{n, m \rightarrow \infty} d\left(x_{n}, x^{*}\right)=0 \text {. }
$$

that is,$x_{n} \rightarrow x^{*}$ as $n \rightarrow \infty$. Now, we will prove that $x^{*}$ is a fixed point of $T$. By (1) we get,

$\mathrm{d}\left(\mathrm{x}^{*}, \mathrm{~T} \mathrm{x}^{*}\right) \leq \alpha\left(\mathrm{x}^{*}, \mathrm{x}_{\mathrm{n}+1}\right) \mathrm{d}\left(\mathrm{x}^{*}, \mathrm{x}_{\mathrm{n}+1}\right)+\beta\left(\mathrm{x}_{\mathrm{n}+1}, \mathrm{~T} \mathrm{x^{* }}\right) \mathrm{d}\left(\mathrm{x}_{\mathrm{n}+1}, \mathrm{x}^{*}\right)$ $=\alpha\left(\mathrm{x}^{*}, \mathrm{x}_{\mathrm{n}+1}\right) \mathrm{d}\left(\mathrm{x}^{*}, \mathrm{x}_{\mathrm{n}+1}\right)+\beta\left(\mathrm{x}_{\mathrm{n}+1}, \mathrm{~T} \mathrm{x}^{*}\right) \mathrm{d}\left(\mathrm{Tx}_{\mathrm{n}}, \mathrm{x}^{*}\right)$

$\leq \alpha\left(\mathrm{x}^{*}, \mathrm{x}_{\mathrm{n}+1}\right) \mathrm{d}\left(\mathrm{x}^{*}, \mathrm{x}_{\mathrm{n}+1}\right)+\beta\left(\mathrm{x}_{\mathrm{n}+1}, \mathrm{~T} \mathrm{x}^{*}\right)\left[\mathrm{a} \mathrm{d}\left(\mathrm{x}_{\mathrm{n}}, \mathrm{x}^{*}\right)+\right.$

$=\alpha\left(\mathrm{x}^{*}, \mathrm{x}_{\mathrm{n}+1}\right) \mathrm{d}\left(\mathrm{x}^{*}, \mathrm{x}_{\mathrm{n}+1}\right)+\beta\left(\mathrm{x}_{\mathrm{n}+1}, \mathrm{~T} \mathrm{x}^{*}\right)\left[\mathrm{ad}\left(\mathrm{x}_{\mathrm{n}}, \mathrm{x}^{*}\right)+\right.$

$\left.\left.\mathrm{Tx}^{*}\right)\right]$

Taking the limit as $n \rightarrow \infty$ and using (3), and the fact that $\lim _{n \rightarrow \infty} \alpha\left(x_{n}, x\right)$ and

finite, we obtain that $\left.d\left(x^{*}, T x^{*}\right) \leq\left[c \lim _{n \rightarrow \infty} \beta\left(x_{n+1}, T x^{*}\right)\right] \quad d\left(x^{*}, T x^{*}\right)\right]$.

$$
\begin{array}{r}
\left.\mathrm{bd}\left(\mathrm{x}_{\mathrm{n}}, \mathrm{Tx}_{\mathrm{n}}\right)+\mathrm{cd}\left(\mathrm{x}^{*}, \mathrm{Tx}^{*}\right)\right] \\
\mathrm{bd}\left(\mathrm{x}_{\mathrm{n}}, \mathrm{x}_{\mathrm{n}+1}\right)+\mathrm{cd}\left(\mathrm{x}^{*},\right.
\end{array}
$$

$\lim _{\mathrm{n} \rightarrow \infty} \beta\left(\mathrm{x}, \mathrm{x}_{\mathrm{n}},\right)$ exist are 13

Suppose that $\mathrm{x}^{*} \neq \mathrm{T} \mathrm{x}^{*}$, having in mind that $\mathrm{c} \lim _{\mathrm{n} \rightarrow \infty} \beta\left(\mathrm{x}_{\mathrm{n}+1}, \mathrm{Tx}^{*}\right)<1$. So, $0<\mathrm{d}\left(\mathrm{x}^{*}, \mathrm{~T} \mathrm{x}^{*}\right) \leq\left[\mathrm{c} \lim _{\mathrm{n} \rightarrow \infty} \beta\left(\mathrm{x}_{\mathrm{n}+1}, \mathrm{Tx}^{*}\right.\right.$ )] $\mathrm{d}\left(\mathrm{x}^{*}, \mathrm{~T} \mathrm{x}^{*}\right)<\mathrm{d}\left(\mathrm{x}^{*}, \mathrm{~T} \mathrm{x}^{*}\right)$.

It is contradiction. This yields that $\mathrm{x}^{*}=\mathrm{T} \mathrm{x^{* }}$.

Let $\mathrm{x}^{* *}$ in $\mathrm{X}$ bee such that $\mathrm{T} \mathrm{x}^{* *}=\mathrm{x}^{* *}$ and $\mathrm{x}^{*} \neq \mathrm{x}^{* *}$. We have,

$0<\mathrm{d}\left(\mathrm{x}^{*}, \mathrm{x}^{* *}\right)=\mathrm{d}\left(\mathrm{Tx}^{*}, \mathrm{Tx}^{* * *}\right)$

$$
\begin{aligned}
& \leq \mathrm{ad}\left(\mathrm{x}^{*}, \mathrm{x}^{* *}\right)+\mathrm{bd}\left(\mathrm{x}^{*}, \mathrm{~T} \mathrm{x}^{*}\right)+\mathrm{cd}\left(\left(\mathrm{x}^{* *}, \mathrm{~T} \mathrm{x}^{* *}\right)\right. \\
& \leq \mathrm{ad}\left(\mathrm{x}^{*}, \mathrm{x}^{* *}\right) .
\end{aligned}
$$

It is a contradiction, so $x^{*}=x^{* *}$. Hence $x^{*}$ is the unique fixed point of $\mathrm{T}$.

\section{RESULT AND DISCUSSION}

Remark 3.1 The assumption (1) in Theorem 2.1 above can be replace by assumption that the mapping $\mathrm{T}$ and the double controlled metric $d$ are continuous. Indeed, when $\mathrm{x}_{\mathrm{n}} \rightarrow \mathrm{x}^{*}, \mathrm{Tx}_{\mathrm{n}} \rightarrow \mathrm{T} \mathrm{x}^{*}$ and hence we have $\lim _{n \rightarrow \infty} d\left(\operatorname{Tx}_{n}, \operatorname{Tx}^{*}\right)=\lim _{n \rightarrow \infty} d\left(\operatorname{Tx}_{n+1}, \operatorname{Tx}^{*}\right)=d\left(x^{*}, T x^{*}\right)$ and hence $\operatorname{Tx}^{*}=x^{*}$.

Theorem 2.1 is illustrated by the following example.

Example 3.1 We endow $X=\{0,1,2\}$ by the following double controlled metric type space $\mathrm{d}: \mathrm{X} \times \mathrm{X} \rightarrow[0, \infty)$ defined by

$\mathrm{d}(0,0)=\mathrm{d}(1,1)=\mathrm{d}(2,2)=0, \mathrm{~d}(0,1)=\mathrm{d}(1,0)=1, \mathrm{~d}(0,2)=\mathrm{d}(2,0)=2 / 5$,

$\mathrm{d}(1,2)=\mathrm{d}(2,1)=6 / 5$

and $\alpha, \beta: X \times X \rightarrow[1, \infty)$ defined by

$\alpha(0,0)=\alpha(1,1)=\alpha(2,2)=1, \alpha(0,2)=\alpha(2,0)=151 / 100, \alpha(0,1)=\alpha(1,0)=6 / 5, \alpha(1,2)=\alpha(2,1)=8 / 5$.

$\beta(0,0)=\beta(1,1)=\beta(2,2)=1, \beta(0,1)=\beta(1,0)=6 / 5, \beta(0,2)=\beta(2,0)=8 / 5, \quad \beta(1,2)=\beta(2,1)=$ $33 / 20$.

Then $\mathrm{d}$ is double controlled metric type space but $\mathrm{d}$ is not a controlled metric type space for the function $\alpha$.

Indeed,

$\mathrm{d}(0,1)=1>247 / 250=\alpha(0,2) \mathrm{d}(0,2)+\alpha(2,1) \mathrm{d}(2,1)$.

Choose function $\mathrm{T}: \mathrm{X} \rightarrow \mathrm{X}$ such that

$\mathrm{T} 0=2$ and $\mathrm{T} 1=\mathrm{T} 2=1$, set

$\mathrm{a}=1 / 6, \mathrm{~b}=1 / 3$ and $\mathrm{c}=1 / 4$.

It is clear that condition (1) is satisfied . In addition (2) holds for each $\mathrm{x}_{0}$ in X. All hypothesis of theorem (2.1) are fulfilled . Here $\mathrm{x}^{*}=1$ is the unique fixed point.

\section{Remark 3.1}

1. In case $\mathrm{b}=\mathrm{c}=0$, we get a result due to Theorem 1 of [ 12 ].

2. In case $a=0, b=c$, we get a result due to Theorem 3 of [ 12 ].

3. In case $\alpha(\mathrm{x}, \mathrm{y})=\beta(\mathrm{x}, \mathrm{y})$, we get a result due to Theorem 8 of [13]. 


\section{International Advanced Research Journal in Science, Engineering and Technology}

Vol. 8, Issue 6, June 2021

DOI: $10.17148 /$ IARJSET.2021.86112

\section{REFERENCE}

1. Bakhtin I.A. "The contraction mapping principle in almost metric spaces.” Funct. Anal., vol. 30, pp. 26 - 37, 1989. [Google Scholar] 2. Czerwik S. "Contraction mapping in b - metric spaces.” Acta Math. Inform. Univ. Ostra., vol. 1,pp. 5 - 11, 1993. [Google Scholar] 3. Abdeljawad T. , Abodayeh K. and Mlaiki N. "On fixed point generalizations to partial b - metric spaces.” J. Comput. Anal. Appl. , vol. 19, pp. $883-891,2015$. [ Google Scholar]

4. Afshari H. , Atapour M. and Aydi H. “Generalized $\alpha-\varphi$ Geraghty multivalued mapping on b - metric spaces endowed with a graph.” TWNS. J. Appl. Eng. Math., vol. 7, 248 - 260, 2017. [ Google Scholar]

5. Alharbi N. , Aydi H. , Felhi A. and Ozel C. , Sahmim S. “ $\alpha$-contraction mappings n rectangular b- metric spaces and application to integral equations.” J. Math. Anal.,v01. 9, 47 -60, 2018. [ Google Scholar]

6. Aydi H. , Karapin E. , Bota M. F. and Mitroviè S. "A fixed point theorem for set - valued quasi cnraction in b- metric space." Fixed Point Theory Appl., vol. 88, 2012. [ Google Scholar] [Cross Ref]

7. Shatanawi W. "Fixed and common fixed point for mapping satisfying some non- linear contraction in b- metric spaces.” J. Math. Anal., vol. 7,pp. 1- 12, 2017. [ Google Scholar]

8. Shatanawi W., Pitea A. and Lazvic V. "Contraction conditions using comparison functions on b- metric spaces." Fixed Point Theory Appl., 2014, 135, 2014. [ Google Scholar ] [ Cross Ref ]

9. Kamran T. , Samreen M. and Radevovic S. “A generalization of b - metric space and some fixed point theorems.” Mathematics vol. 5,pp. 1- 17, 2017. [ Google Scholar ] [Cross Ref]

10. Huang H. , Den G. and Radevovic S. "Fixed point theorems in b - metric spaces with applications to differential equations." J. Fixed Point Theory Appl., 2018. [ Google Scholar] [Cross Ref ]

11. Mlaiki N. , Aydi H. , Souayah N. and Abdeljawad T. "Controlled metric type space and the related contractions principal." Mathematics vol. 6, 194, 2016. [ Google Scholar] [Cross Ref]

12. Abdeljawad T. , Mlaiki N. , Aydi H. and Souayah N. "Double controlled metric type spaces and some fixed point results." Mathematics vol. 6, $12,3202018$.

13. Ahmad J., Al- Mazrooei A.E. , Aydi H . and Sen M.D.L. “ On fixed point results in controlled metric spaces.” J. Funct. Spaces, 2020, 2020, Article ID 2108167.

14. Kannan R. "Some results on fixed points.” Bull. Calcutta Math. Soc.,vol. 60, pp. 71- 76, 1968.[ Google Scholar]

15. Reich, S. "Fixed point of contractive functions." Bollettino dell Unione Mathematica Italiana, 4,pp. 26 - 42, 1972. [ Google Scholar]

16. Reich, S. "Kannan's fixed point theorem.” Bollettino dell Unione Mathematica Italiana, vol. 4, pp. 1 - 11, 1971. [ Google Scholar] 